\title{
An application of fuzzy TOPSIS for identifying and analyzing black spots suburban areas
}

\author{
Ali Wersi Qazvini ${ }^{a^{*}}$, Hassan Javanshir $^{\mathrm{b}}$ and Seyed Mohammad Seyedhossenic
}

${ }^{a}$ Department of Transportation Planning and Traffic Engineering, South Tehran Branch, Islamic Azad University, Tehran, Iran

${ }^{b}$ Department of Industrial Engineering, South Tehran Branch, Islamic Azad University, Tehran, Iran

cDepartment of Industrial Engineering, Iran University of Science and Technology, Tehran, Iran

\section{H R O N I C L E}

Article history:

Received January 5, 2016

Received in revised format April

15,2016

Accepted June 27, 2016

Available online

June 28, 2016

Keywords:

Fuzzy TOPSIS

Black spots

Road accident

\section{Introduction}

Statistics show that for every 10 thousand cars in the world about 9 people are killed in road incidents, while in Iran the rate of fatality is about 37 , which is higher than average fetal accidents in the world. Basically crash refers to a situation in which several parameters, including human factors, operating the vehicle as well as environmental issues are involved. Incidents normally influences significantly on growth domestic product of every country. The issue of accident and casualties have absorbed significant attentions of governmental agencies and they are repeatedly requested to execute appropriate planning to reduce the burden of casualties. Nowadays, traffic safety is one of the primary principles of traffic engineering and transport planning. Road accidents has been one of the major problems that cause human losses, social and economic challenges. The first step to improve road traffic safety is to identify black spots, which preserve high potential of road accidents. Therefore, identifying, analyzing, prioritizing and refining these places play important role in improving transportation safety.

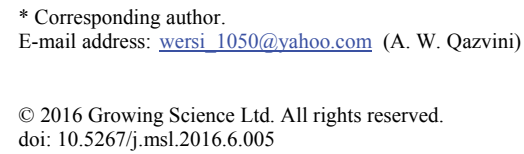


With the development of road safety improvement, different actions are performed to identify two major objectives, namely high-accident locations and the locations that have the greatest potentials to reduce accidents. In Iran, 52\% of the incidents are human related, 30\% of them are associated with roads and $12 \%$ of them are related to vehicles. Therefore, any action on reducing road black spots could improve the quality of the roads. Cirović and Pamučar (2013) presented a modeling of the Adaptive Neuro Fuzzy Inference System (ANFIS), which supports the process of choosing which level crossings ought to receive an investment of safety equipment in rail way transportation. They used ANFIS model, which was trained using a method of fuzzy multi-criteria decision making and fuzzy clustering techniques. The proposed model was trained with the experiential knowledge of these experts and examined on a selection of rail crossings in the Belgrade area regarding an investment of safety equipment.

Chen et al. (2015) presented an improved entropy TOPSIS-RSR method to perform the road safety risk evaluation process from an overall perspective, based on a composite Road Safety Risk Index (RSRI). They reported the feasibility of applying the model as a valuable tool for road safety policymakers to decision-making activities. Bao et al. (2012) proposed an improved hierarchical fuzzy TOPSIS model to integrate the multilayer Safety performance indicators (SPIs) into one overall index by including experts' knowledge. They used the number of road fatalities per million inhabitants as a relevant reference and provided an intelligent decision support system to make an assessment on the road safety performance for a case study of European countries. The proposed study handled experts' linguistic expressions and used the layered hierarchy of the indicators.

\section{The proposed study}

This paper applies fuzzy Technique for Order Preference by Similarity to Ideal (TOPSIS) for ordering different black spots in a case study of highway between two cities of Qazvin and Saveh, Iran. The study performs the ranking based on two categories of tangent distance and horizontal curve. TOPSIS method has been extensively used for transportation planning (Mirmohammadi et al., 2013). TOPSIS method uses different criteria for ranking various alternatives. The criteria must be first ranked using an MCDM techniques such as Analytical hierarchy method.

\subsection{The AHP method}

The nature of multiple criteria decision making (MCDM) is to make a fair decision making and to remove the false or misleading decision making as much as possible. Therefore, a decision process requires to be supplied by an effective and low risk technique. Moreover, each problem can be evaluated independently by dis-integrating the decision problem into a hierarchy, which reduces probable errors. Analytical hierarchy process (AHP), proposed by Saaty (1980), is an MCDM technique that addresses how to detect the relative importance of a set of activities in an MCDM problem and normally is implemented to overcome the uncertainty. When we use AHP, we consider judgments on tangible and intangible criteria and determine the weights of each criterion (Badri, 2001). Normally, the most important advantage of AHP is the hierarchy structure of the model (Wu et al., 2012; Khajeh, 2010). The first step in AHP is to create some levels of hierarchy of interrelated decision criteria and alternatives according to different objectives. The next step is to compare the alternatives and criteria by prioritizing to determine the relative importance of criteria in each level of hierarchy (Amiri, 2010).

AHP in recent decades has been increasingly implemented to solve various types of MCDM problems. Some people have mentioned AHP as an effective method in varieties of problems including road accidents (Hermans et al., 2008; Najib et al., 2012; Agarwal et al. 2013). AHP methodology follows the steps below to find relative importance degree of criteria:

Step 1 - Prepare the pair wise comparison matrix A using the ratio scales given in Table 1. 
Table 1

The ratio scale and definition of AHP

\begin{tabular}{clcl}
\hline Intensity of importance & Definition & Intensity of & Definition \\
\hline 1 & Equally important & 7 & Very strong \\
3 & Moderately important & 9 & Extremely more \\
5 & Strongly more important & $2,4,6,8$ & Intermediate more \\
\hline
\end{tabular}

Step 2 - Let $C_{1}, C_{2}, \ldots . ., C_{n}$ be the set of elements, where $a_{i j}$ is a quantified judgment on pair of elements $C_{i,}, C_{j .}$ the matrix A as follows;

$$
A=\left[a_{i j}\right]=\left[\begin{array}{cccc}
1 & a_{12} & \ldots & a_{1 n} \\
\frac{1}{a_{12}} & 1 & \ldots & a_{2 n} \\
\vdots & \vdots & \vdots & \vdots \\
\frac{1}{a_{1 n}} & \frac{1}{a_{2 n}} & \ldots & 1
\end{array}\right]
$$

where, $a_{i j}=1$ and $a_{j i}=\frac{1}{a_{i j}}, i . j=1,2, \ldots, n$

In matrix $A$, the problem is to determine a set of numerical weights $W_{1}, W_{2}, \ldots ., W_{n}$ in front of $n$ element $C_{1}, C_{2}, \ldots, C_{n}$. If $A$ is a consistency matrix, then the relation between weights and judgments are given by $a_{i j}=\frac{W_{j}}{W_{i}}$, for $(i, j=1,2,3, \ldots ., n)$. The largest Eigen-value $\lambda_{\max }$ would be suggested by Saaty (1980) as;

$$
\lambda_{\max }=\sum_{j=1}^{n} a_{i j} \frac{W_{j}}{W_{i}}
$$

Let $A$ be the consistency matrix, then eigenvector $X$ can be expressed as follows,

$$
\left(A-\lambda_{\max } I\right) X=0
$$

Therefore, the consistency index (C.I.) and random index (R.I.) represent the consistency ratio (C.R.). The consistency index and consistency rate are as follows,

$$
\text { C.I. }=\frac{\lambda_{\max }-n}{n-1} \text {, and } C . R .=\frac{C . I}{R . I}
$$

A number 0.1 is an accepted upper limit of C.R. If the final consistency ration is more than this value, the evaluation process has to be repeated to improve consistency.

\subsection{Preliminary of fuzzy approach}

Fuzzy set theory is a class of objects with grades of membership applied to model vagueness and to cope with uncertainty by a membership function (Kutlu \& Ekmekçioğlu, 2012). It uses linguistic terms to present decision makers preferences.

Definition 1 - A fuzzy set $\tilde{\mathrm{A}}$ in a universe of discourse $X$ is explained by a membership function $\mu_{-}(x)$. It joints with each element $x$ in $X$, a real number in the interval $[0,1]$. The function value $\mu_{-}(x)$ is designated the grade of membership of $x$ in $\tilde{A}$. 
This study focuses on triangular fuzzy numbers. A triangular fuzzy number $\tilde{\mathrm{A}}$ is defined as $\left(a_{1}, a_{2}, a_{3}\right)$ , where $a_{3}>a_{2}>a_{1}$. The following equation displays mathematical form of triangular fuzzy number and Fig. 1 also shows a triangular number.

$$
\mu_{a}(x)=\left\{\begin{array}{cc}
0, & x \leq a_{1} \\
\frac{x-a_{1}}{a_{2}-a_{1}}, & a_{1}<x \leq a_{2} \\
\frac{a_{3}-x}{a_{3}-a_{2}}, & a_{2}<x \leq a_{3} \\
0, & x>a_{3}
\end{array}\right.
$$

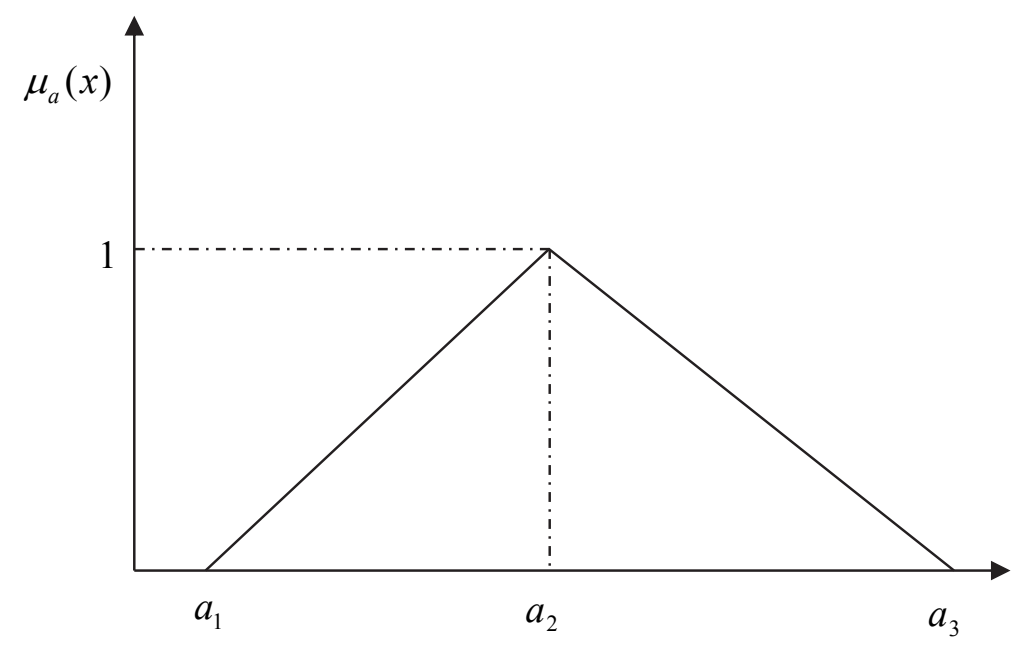

Fig. 1. Triangular number

Definition 2 - Let $a=\left(a_{1}, a_{2}, a_{3}\right)$ and $b=\left(b_{1}, b_{2}, b_{3}\right)$ be two triangular fuzzy numbers, the distance between them is computed as Eq. (5) as follows,

$$
d \tilde{a}, \tilde{b})=\sqrt{\frac{1}{3}\left[\left(a_{1}-b_{1}\right)^{2}+\left(a_{2}-b_{2}\right)^{2}+\left(a_{3}-b_{3}\right)^{2}\right]}
$$

In addition, the Table 2 shows the operational equations of the two triangular fuzzy numbers.

\section{Table 2}

Basic equations of the two triangular fuzzy numbers

\begin{tabular}{ll}
\hline Operational law & Equations \\
\hline Addition & $(a+b)=\left(a_{1}+b_{1}, a_{2}+b_{2}, a_{3}+b_{3}\right)$ \\
Subtraction & $(a-b)=\left(a_{1}-b_{1}, a_{2}-b_{2}, a_{3}-b_{3}\right)$ \\
Multiplication & $(a \times b)=\left(a_{1} \times b_{1}, a_{2} \times b_{2}, a_{3} \times b_{3}\right), k(a)=\left(k a_{1}, k a_{2}, k a_{3}\right)$ \\
Division & $(a \div b)=\left(a_{1} \div b_{1}, a_{2} \div b_{2}, a_{3} \div b_{3}\right)$ \\
Inverse & $\left(a_{1}, a_{2}, a_{3}\right)^{-1}=\left(\frac{1}{a_{3}}, \frac{1}{a_{2}}, \frac{1}{a_{1}}\right)$ \\
\hline
\end{tabular}

\subsection{The fuzzy TOPSIS method}

TOPSIS as a classical multiple criteria decision making methods was originally proposed by Hwang and Yoon (1981) to analyze alternative solutions among each criterion and eventually to detect the most 
efficient alternatives. The TOPSIS algorithm is associated with the shortest distance from the positive ideal solution (PIS) and farthest from negative ideal solution (NIS). However, often for decision makers somehow it is difficult to assign a precise evaluation rating to an alternative. The advantage of fuzzy approach is to overcome the vagueness of human judgments and to receive relative importance of attributes (Yang \& Hung, 2007). The fuzzy TOPSIS procedure is introduced here (Awasthi et al., 2011);

Step 1 - Assignment of rating to different criteria and various alternatives. Assume there are $j$ possible candidates, $A=\left\{A_{1}, A_{2}, \ldots . . A_{j}\right\}$, which are assessed against $m$ criteria, $C=\left\{C_{1}, C_{2}, \ldots . C_{m}\right\}$. The criteria weights are given by $w_{i}=\{i=1,2, \ldots ., m\}$. The performance ratings of each decision maker $D_{k}(k=1,2, \ldots, K)$ for each alternative $A_{j}(j=1,2, \ldots, n)$ respecting to criteria $C_{i}(i=1,2, \ldots, m)$ are also given by $\tilde{R}_{k}=\tilde{x}_{i j k}(i=1,2, \ldots, m ; j=1,2, \ldots ., n ; k=1,2, \ldots ., k)$ with membership function $\mu_{\tilde{R}_{k}}(x)$

Step 2 - Calculate aggregate fuzzy rating for the alternatives and the criteria where the fuzzy rating of all decision maker about criteria are given as triangular fuzzy numbers $\tilde{R}_{k}=\left(a_{k}, b_{k}, c_{k}\right)$, $k=1,2, \ldots . ., K$, and the aggregated fuzzy rating is obtained by $\tilde{R}=(a, b, c), k=1,2, \ldots . ., K$, where

$$
a=\min _{k}\left\{a_{k}\right\}, \quad b=\frac{1}{k} \sum_{k=1}^{K} b_{k}, \quad c=\max _{k}\left\{c_{k}\right\} .
$$

When the fuzzy rating and importance weight of the $k^{t h}$ decision maker are $\tilde{x}_{i j k}=\left(a_{i j k}, b_{i j k}, c_{i j k}\right)$ and $\tilde{w}_{i j k}=\left(w_{j k 1}, w_{j k 2}, w_{j k 3}\right), i=1,2, \ldots ., m, j=1,2, \ldots . ., n$ respectively, the integrated fuzzy ratings $\left(\tilde{x}_{i j}\right)$ of alternatives with in terms of each criterion are given by $\tilde{x}_{i j}=\left(a_{i j}, b_{i j}, c_{i j}\right)$ where

$$
a_{i j}=\min _{k}\left\{a_{i j k}\right\}, \quad b_{i j}=\frac{1}{k} \sum_{k=1}^{K} b_{i j k}, \quad c_{i j}=\max _{k}\left\{c_{i j k}\right\} .
$$

The aggregated fuzzy weights $\left(\tilde{w}_{i j}\right)$ of each criterion are measured as $\tilde{w}_{j}=\left(w_{j 1}, w_{j 2}, w_{j 3}\right)$ where

$$
w_{j 1}=\min _{k}\left\{w_{1 j k}\right\}, \quad w_{j 2}=\frac{1}{k} \sum_{k=1}^{K} w_{j k 2}, \quad w_{j 3}=\underset{k}{\max }\left\{c_{j k 3}\right\} .
$$

Step 3 - Compute the fuzzy decision matrix

The fuzzy decision matrix for alternatives $(\tilde{D})$ and criteria $(\tilde{W})$ is arranged as follows;

$$
\begin{aligned}
\tilde{D} & =\left[\begin{array}{cccc}
\tilde{x}_{11} & \tilde{x}_{12} & \ldots & \tilde{x}_{1 n} \\
\tilde{x}_{21} & \tilde{x}_{22} & \ldots . & \tilde{x}_{2 n} \\
\ldots & \ldots & \ldots & \ldots \\
\tilde{x}_{m 1} & \tilde{x}_{m 1} & \ldots & \tilde{x}_{m n}
\end{array}\right], \quad i=1,2, \ldots ., m, \quad j=1,2, \ldots . ., n \\
\tilde{W} & =\left(\tilde{w}_{1}, \ldots . ., \tilde{w}_{n}\right) .
\end{aligned}
$$

Step 4 - The raw data are normalized using a linear scale transforming to bring the different criterion scales on to a comparable scale. The normalized fuzzy decision matrix is given by

$$
\tilde{R}=\left[\tilde{r}_{i j}\right]_{m \times n}, \quad i=1,2, \ldots . ., m, \quad j=1,2, \ldots . ., n,
$$

where

$$
\tilde{r}_{i j}=\left(\frac{a_{i j}}{c_{j}^{*}}, \frac{b_{i j}}{c_{j}^{*}}, \frac{c_{i j}}{c_{j}^{*}}\right) \text {, and } c_{j}^{*}=\max _{i} c_{i j} \text { (benefit criteria) } \tilde{r}_{i j}=\left(\frac{a_{j}^{-}}{c_{i j}}, \frac{a_{j}^{-}}{b_{i j}}, \frac{a_{j}^{-}}{a_{i j}}\right) \text {, an } a_{j}^{-}=\underset{i}{\min } a_{i j} \text { (cost criteria) }
$$


Step 5 - Measurement of weighted normalized matrix. The weighted normalized matrix $\tilde{V}$ for criteria is measured by multiplication of the weights $\left(\tilde{w}_{j}\right)$ of evaluation criteria with the normalized fuzzy decision matrix $\tilde{r}_{i j}$;

$$
\tilde{v}=\left[\tilde{v}_{i j}\right]_{m \times n}, \quad i=1,2, \ldots . . m, \quad j=1,2, \ldots . ., n \text { where } \tilde{v}_{i j}=\tilde{r}_{i j}(.) \tilde{w}_{j} .
$$

Step 6 - Arrangement of the FPIS (fuzzy positive ideal solution) and FNIS (fuzzy negative ideal solution) for alternatives. FPIS and FNIS as follows;

$$
\begin{aligned}
& A^{*}=\left(\tilde{v}_{1}^{*}, \cdots, \tilde{v}_{n}^{*}\right), \text { where } \tilde{v}_{j}^{*}=\max _{i}\left\{v_{i j 3}\right\}, i=1,2, \ldots, m \text { and } j=1,2, \ldots, n \\
& A^{*}=\left(\tilde{v}_{1}^{-}, \cdots, \tilde{v}_{n}^{-}\right), \text {where } \tilde{v}_{j}^{-}=\min _{i}\left\{v_{i j 1}\right\}, i=1,2, \ldots ., m \text { and } j=1,2, \ldots, n
\end{aligned}
$$

Step7 - measure the distance of each alternative from FPIS and FNIS. The distance $\left(d_{i}^{*}, d_{i}^{-}\right)$ of each weighted alternative $i=1,2, \ldots ., m$ from the FPIS and FNIS is as follows,

$$
\begin{array}{ll}
d_{i}^{*}=\sum_{j=1}^{n} d_{v}\left(\tilde{v}_{i j}, \tilde{v}_{j}^{-}\right), & i=1,2, \ldots, m \\
d_{i}^{-}=\sum_{j=1}^{n} d_{v}\left(\tilde{v}_{i j}, \tilde{v}_{i j}^{-}\right), & i=1,2, \ldots, m
\end{array}
$$

where $d_{v}(\tilde{a}, \tilde{b})$ is the distance measurement between two fuzzy numbers $\tilde{a}$ and $\tilde{b}$

Step 8 - measure the closeness coefficient $\left(c c_{i}\right)$ of each alternative. The closeness coefficient represents the distances to the fuzzy positive ideal solution and fuzzy negative ideal solution, simultaneously. The closeness coefficient of each alternative is as follows,

$$
c c_{i}=\frac{d_{i}^{-}}{d_{i}^{-}+d_{i}^{*}}, \quad i=1,2, \ldots, m
$$

Step 9 - Rank the alternatives

\section{The results}

This paper applies fuzzy TOPSIS for ordering different black spots in a case study of highway between two cities of Qazvin and Saveh, Iran. The study performs the ranking based on two categories of tangent distance and horizontal curve. The ranking is performed according to Roadway width $\left(\mathrm{C}_{\mathrm{RW}}\right)$, Shoulder width $\left(\mathrm{C}_{\mathrm{SW}}\right)$, Traffic volume $\left(\mathrm{C}_{\mathrm{NW}}\right)$, Percentage of vehicle $\left(\mathrm{C}_{\mathrm{PV}}\right)$, Load and utility $\left(\mathrm{C}_{\mathrm{LU}}\right)$, Network access $\left(\mathrm{C}_{\mathrm{NA}}\right)$, The number of horizontal curves $\left(\mathrm{C}_{\mathrm{NC}}\right)$ and Equivalent Property Damage Only $\left(\mathrm{C}_{\mathrm{EPDO}}\right)$. Table 3 and Table 4 present the linguistic terms, associated fuzzy numbers and the values assigned to different criteria, respectively.

Table 3

Definition of linguistic terms

\begin{tabular}{lclc}
\hline Importance & Fuzzy number & Importance & Fuzzy number \\
\hline Very low (VL) & $(0,0,0.2)$ & Medium-high & $(0.5,0.65,0.8)$ \\
Low & $(0.1,0.2,0.3)$ & High & $(0.7,0.8,0.9)$ \\
Medium-low (ML) & $(0.2,0.35,0.5)$ & Very high & $(0.8,1,1)$ \\
Medium (M) & $(0.4,0.5,0.6)$ & & \\
\hline
\end{tabular}


Table 4

The weights given to different criteria

\begin{tabular}{cccc}
\hline Criterion & $D_{P}$ & $D_{H}$ & $D_{R}$ \\
\hline$C_{\mathrm{RW}}$ & $\mathrm{VH}$ & $\mathrm{VH}$ & $\mathrm{VH}$ \\
$\mathrm{C}_{\mathrm{LU}}$ & $\mathrm{H}$ & $\mathrm{H}$ & $\mathrm{VH}$ \\
$\mathrm{C}_{\mathrm{SW}}$ & $\mathrm{MH}$ & $\mathrm{MH}$ & $\mathrm{H}$ \\
$\mathrm{C}_{\mathrm{PW}}$ & $\mathrm{MH}$ & $\mathrm{H}$ & $\mathrm{H}$ \\
$\mathrm{C}_{\mathrm{EPDO}}$ & $\mathrm{VH}$ & $\mathrm{MH}$ & $\mathrm{MH}$ \\
$\mathrm{C}_{\mathrm{NA}}$ & $\mathrm{VH}$ & $\mathrm{MH}$ & $\mathrm{MH}$ \\
$\mathrm{C}_{\mathrm{NW}}$ & $\mathrm{H}$ & $\mathrm{VH}$ & $\mathrm{H}$ \\
\hline
\end{tabular}

The proposed study of this paper has detected 21 different black spots in the high way of Qazvin-Saveh located in Iran. Each alternative is denoted with a letter A and a number, which indicates the distance from the origin. For instance, A15 represents a black spot which is 15 kilometer away from the city of Qazvin. As mentioned, the study has performed the ranking based on two categories of tangent distance and horizontal curve and the results are compared with the frequencies of accidents. Table 5 demonstrates the results in terms of tangent distance. In addition, Table 6 shows the results based on horizontal crave.

Table 5

The summary of the ranking black spots based on tangent distance

\begin{tabular}{|c|c|c|c|c|}
\hline \multirow[t]{2}{*}{$N$} & \multicolumn{2}{|c|}{ FTOPSIS } & \multicolumn{2}{|c|}{ Frequency of incidents $\left(F_{t}\right)$} \\
\hline & $A_{i}$ & $\left(\mathrm{ccc}_{\mathrm{i}}\right)$ & $A_{i}$ & $\mathrm{~F}_{\mathrm{t}}$ \\
\hline 1 & A15 & 0.471 & $\mathrm{~A} 42$ & 19 \\
\hline 2 & A57 & 0.455 & A15 & 18 \\
\hline 3 & A42 & 0.436 & A57 & 18 \\
\hline 4 & A37 & 0.432 & A37 & 16 \\
\hline 5 & A53 & 0.422 & A12 & 15 \\
\hline 6 & A56 & 0.416 & A56 & 14 \\
\hline 7 & A25 & 0.378 & A53 & 13 \\
\hline 8 & A48 & 0.367 & A89 & 12 \\
\hline 9 & A24 & 0.365 & A88 & 12 \\
\hline 10 & A30 & 0.362 & A24 & 11 \\
\hline 11 & A12 & 0.334 & A48 & 10 \\
\hline 12 & A59 & 0.328 & A25 & 9 \\
\hline 13 & A89 & 0.326 & A59 & 9 \\
\hline 14 & A7 & 0.317 & A32 & 9 \\
\hline 15 & $\mathrm{~A} 43$ & 0.315 & $\mathrm{~A} 43$ & 9 \\
\hline 16 & $\mathrm{~A} 20$ & 0.309 & $\mathrm{~A} 30$ & 8 \\
\hline 17 & A52 & 0.298 & A7 & 7 \\
\hline 18 & A88 & 0.296 & A52 & 7 \\
\hline 19 & $\mathrm{~A} 32, \mathrm{~A} 33$ & 0.295 & $\mathrm{~A} 33$ & 7 \\
\hline 20 & A38 & 0.294 & $\mathrm{~A} 38$ & 6 \\
\hline 21 & A58 & 0.289 & A58 & 4 \\
\hline
\end{tabular}

Table 6

The results of the implementation of FTOPSIS for ranking 21 alternatives based on horizontal crave

\begin{tabular}{ccccc}
\hline $\mathrm{N}$ & \multicolumn{2}{c}{ FTOPSIS } & \multicolumn{2}{c}{ Frequency of incidents $\left(\mathrm{F}_{\mathrm{t}}\right)$} \\
\cline { 2 - 5 } & $A_{i}$ & $\mathrm{~F}_{\mathrm{t}}$ & $A_{i}$ & $\mathrm{~F}_{\mathrm{t}}$ \\
\hline 1 & $\mathrm{~A} 45$ & 0.491 & $\mathrm{~A} 45$ & 22 \\
2 & $\mathrm{~A} 64$ & 0.452 & $A 10$ & 15 \\
3 & $\mathrm{~A} 10$ & 0.414 & $\mathrm{~A} 64$ & 12 \\
4 & $\mathrm{~A} 81$ & 0.413 & $\mathrm{~A} 81$ & 12 \\
5 & $\mathrm{~A} 62, \mathrm{~A} 66$ & 0.403 & $\mathrm{~A} 22, \mathrm{~A} 27$ & 8 \\
6 & $\mathrm{~A} 16, \mathrm{~A} 23$ & 0.397 & $\mathrm{~A} 62, \mathrm{~A} 66$ & 7 \\
7 & $\mathrm{~A} 22, \mathrm{~A} 27$ & 0.373 & $\mathrm{~A} 23$ & 6 \\
8 & $\mathrm{~A} 83$ & 0.364 & $\mathrm{~A} 16$ & 5 \\
9 & $\mathrm{~A} 17$ & 0.359 & $\mathrm{~A} 83$ & 5 \\
\hline
\end{tabular}




\section{Conclusion}

In this paper, we have presented an empirical investigation to prioritize black spots in one of Iranian highways. The study has implemented fuzzy TOPSIS to rank different alternatives according to various criteria. The study results of the ranking in two categories of tangent distance and horizontal crane have been compared with frequencies of incidents and the results were somehow in harmony. We hope the results of the survey could help different governmental agencies allocate budget more appropriately.

\section{Acknowledgement}

The authors would like to thank the anonymous referees for constructive comments on earlier version of this paper.

\section{References}

Agarwal, P. K., Patil, P. K., \& Mehar, R. (2013). A methodology for ranking road safety hazardous locations using analytical hierarchy process.Procedia-Social and Behavioral Sciences, 104, 1030-1037.

Amiri, M. P. (2010). Project selection for oil-fields development by using the AHP and fuzzy TOPSIS methods. Expert Systems with Applications, 37(9), 6218-6224.

Awasthi, A., Chauhan, S. S., \& Goyal, S. K. (2010). A fuzzy multi criteria approach for evaluating environmental performance of suppliers. International Journal of Production Economics, 126(2), 370378.

Badri, M. A. (2001). A combined AHP-GP model for quality control systems. International Journal of Production Economics, 72(1), 27-40.

Bao, Q., Ruan, D., Shen, Y., Hermans, E., \& Janssens, D. (2012). Improved hierarchical fuzzy TOPSIS for road safety performance evaluation.Knowledge-based Systems, 32, 84-90.

Chen, F., Wang, J., \& Deng, Y. (2015). Road safety risk evaluation by means of improved entropy TOPSISRSR. Safety Science, 79, 39-54.

Ćirović, G., \& Pamučar, D. (2013). Decision support model for prioritizing railway level crossings for safety improvements: Application of the adaptive neuro-fuzzy system. Expert Systems with Applications, 40(6), 2208-2223.

Hermans, E., Van den Bossche, F., \& Wets, G. (2008). Combining road safety information in a performance index. Accident Analysis \& Prevention,40(4), 1337-1344.

Hwang, C. L. \& Yoon, K. (1981). Multiple Attribute Decision Making: Methods and Applications. New york: Springer- Verlag.

Khajej, M. (2010). Water conservation in Kuwait: a fuzzy analysis approach. Journal of Industrial Engineering International, 6(10), 90-105.

Kutlu, A. C., \& Ekmekçioğlu, M. (2012). Fuzzy failure modes and effects analysis by using fuzzy TOPSISbased fuzzy AHP. Expert Systems with Applications, 39(1), 61-67.

Mirmohammadi, F., Khorasani, G., Tatari, A., Yadollahi, A., Taherian, H., Motamed, H., ... \& Maleki Verki, M. (2013). Investigation of road accidents and casualties factors with MCDM methods in Iran. Journal of American Science, 9(7), 11-20.

Najib, L., Abdullah, L., Abdullah, I., \& Salleh, Z. (2012). Weights of road accident causes using analytic hierarchy process. ARPN Journal of Science and Technology, 2, 39-44.

Saaty, T. (1980). The analytic hierarchy process. New york: Mc Grae-Hill.

Wu, H. Y., Chen, J. K., Chen, I., \& Zhuo, H. H. (2012), Ranking universities based on performance evaluation by a hybrid MCDM model. Measurement, 45(5), 856-880.

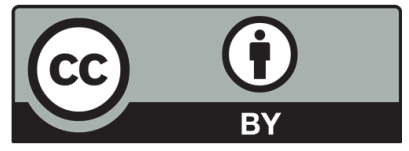

(C) 2016 by the authors; licensee Growing Science, Canada. This is an open access article distributed under the terms and conditions of the Creative Commons Attribution (CC-BY) license (http://creativecommons.org/licenses/by/4.0/). 